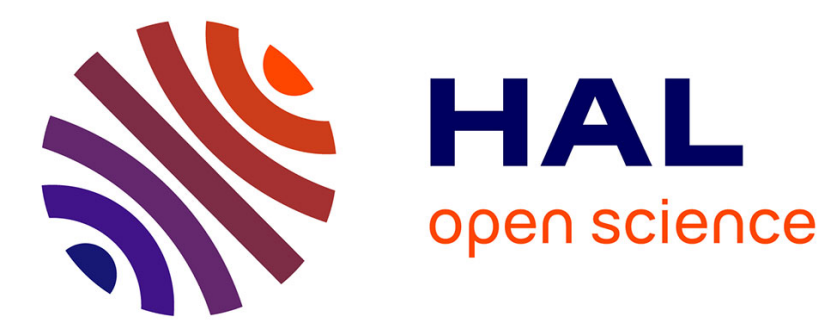

\title{
Land Arts. Les fabriques spatiales de l'art contemporain
}

Anne Volvey

\section{To cite this version:}

Anne Volvey. Land Arts. Les fabriques spatiales de l'art contemporain. Travaux de l'Institut de Géographie de Reims, 2007, 129-130, pp.3-25. halshs-00426783

\section{HAL Id: halshs-00426783 \\ https://shs.hal.science/halshs-00426783}

Submitted on 27 Oct 2009

HAL is a multi-disciplinary open access archive for the deposit and dissemination of scientific research documents, whether they are published or not. The documents may come from teaching and research institutions in France or abroad, or from public or private research centers.
L'archive ouverte pluridisciplinaire HAL, est destinée au dépôt et à la diffusion de documents scientifiques de niveau recherche, publiés ou non, émanant des établissements d'enseignement et de recherche français ou étrangers, des laboratoires publics ou privés. 
Travaux de l'Institut de Géographie de Reims,

$\mathrm{n}^{\circ} 129-130,2008-$ pp. 3-25

\begin{tabular}{c||l}
\multicolumn{1}{c||}{ LAND ARTS } & $\begin{array}{l}\text { Anne } \\
\text { VOLVEY }\end{array}$ \\
Les fabriques spatiales & \\
de l'art contemporain & $\begin{array}{l}\text { Maître de Conférences en géographie à } \\
\text { l'universitéd'Artois } \\
\text { Membre d'Ehgo, } \\
\text { UMR 8504 Géographie-Cités } \\
\text { anne.volvey@univ-artois.fr }\end{array}$ \\
\end{tabular}

Mots-clés : art contemporain ; géographie ; Land art ; spatialité ; chôra ; land ; land claiming ; terrain ; cartographie ; carte

Résumé - Témoignage du renforcement et du renouvellement d'une pluridisciplinarité autour de l'art contemporain, Spatialités de l'art ouvre plusieurs pistes pour penser la spatialité de l'œuvre d'art contemporaine. Ce collectif de textes opère une série de décentrements par rapport aux référents plus habituels de cette pluridisciplinarité : les auteur(e)s choisissent leurs exemples dans la danse et les installations plutôt que dans la littérature et la peinture ; ils s'intéressent aux pratiques artistiques, tout particulièrement au travail de terrain et à la cartographie, plus qu'aux objets d'art qu'ils placent dans la perspective de la pratique ; ce faisant, ils examinent de nouveaux objets, la carte, et réexaminent de manière critique les anciens, le paysage ; ils conçoivent la dimension politique de l'œuvre d'art dans la perspective d'une logique d'action et non de représentation.

Une partie du texte d'introduction de ce numéro se veut une contribution à la construction de l'intelligibilité de la spatialité de l'art contemporain. Elle présente, du point de vue de la géographie contemporaine, les éléments théoriques d'une compréhension du changement de matrice disciplinaire opéré par le Land Art états-unien, et s'appuie tout particulièrement sur la pratique, les objets et les citations de Dennis Oppenheim et de Christo et Jeanne-Claude. En rechargeant de sens la notion de land (dans Land Art), elle trouve les fondements d'un schème d'intelligibilité de la dimension spatiale des arts contemporains, dans leurs pratiques, leurs objets et leurs visées. Une deuxième partie place les textes rassemblés dans le numéro, dans cette perspective théorique pour donner à voir un ensemble de pratiques, objets, disciplines et paradigmes amenés par ce renouvellement pluridisciplinaire. Ce faisant, elle démontre que l'art n'est pas simplement objet construit de la pluridisciplinarité, mais qu'il concourt à sa construction au titre reconnu de discipline (voire science) de l'espace. 
Keywords: contemporary art; geography; Land Art; spatiality; chôra; land; land claiming; fieldwork; mapping; map

Abstract - Spatialities of Art contributes as a collection to the recent reinforcement and renewal of the pluridisciplinarity around Contemporary Art. It aims at considering new ways of thinking Contemporary Art and opens up possibilities that were not addressed in previous approaches: authors engage Dance and Installations rather than limit themselves to Literature and Painting; they focus on art practices, mainly fieldworking and mapping, rather than on objects of art - which are rather replaced within the perspective of art practices. In so doing, they investigate new objects, the map and the territory, and critically examine more classical ones, such as the landscape ; they understand the political dimension of the work of art within a politics of action rather than within a politics of representation.

This paper more specifically addresses the spatial dimension of Contemporary Arts and aims at renewing its understanding. Speaking from a geographical standpoint, it uses the American Land Art, mainly resorting to Dennis Oppenheim's and, Christo and Jeanne-Claude's practices, objects and texts as a new matrix for a theoretical understanding of Art. Reloading and expanding the signification of land (in Land Art), it grounds a schema of intelligibility of the spatial dimension of Contemporary Arts in their practices, objects and designs. The second part replaces the collection of papers within this theoretical framework and shed light on the set of practices, objects, disciplines and paradigms put forward by this pluridisciplinary renewal. It thus demonstrates that Art is not simply a constructed object of pluridisciplinarity, but that it participates in it as an acknowledged discipline (even science) of space.

Spatialités de l'art est un numéro spécial thématique qui témoigne des et participe aux renforcement et renouvellement d'une pluridisciplinarité entre art, esthétique, histoire de l'art, architecture et géographie, finalement peu élaborée jusqu'à aujourd'hui - renforcement et renouvellement liés à l'évolution des pratiques contemporaines des arts, et tout particulièrement de leur dimension spatiale. En tant que telle, dans la mesure où elle construit l'intelligibilité de la spatialité de l'œuvre de l'art contemporaine, et que, ce faisant, elle tente d'articuler des cultures disciplinaires, de croiser des notions et des procédures cognitives, cette pluridisciplinarité participe du tournant spatial de la pensée contemporaine.

Cette introduction du numéro se veut à la fois proposition théorique et présentation des textes d'auteurs ici rassemblés. Construite dans le champ de la géographie, cette proposition élabore une intelligibilité de la spatialité des arts contemporains. Elle trouve son principe dans une problématisation renouvelée de la spatialité du Land Art états-unien, problématisation qui prend au sérieux et recharge sémantiquement la notion de land pour l'étendre aux pratiques artistiques contemporaines. Quant à la présentation des textes, elle est en grande partie placée dans la perspective ainsi dégagée, et s'organise autour de trois thèmes clés : l'enjeu foncier, le cartographique dans l'art contemporain, l'art contemporain comme science de l'espace. Cette présentation thématique recoupe tout en la resserrant l'organisation en chapitres du numéro, elle en explicite les fondements théoriques et paradigmatiques (au sens strict du terme), mais, de par son orientation, elle ne saurait épuiser la richesse de la collection. 


\section{Spatialités de l'art dans la perspective d'une pluridisciplinarité renouvelée}

Ce numéro thématique témoigne d'un renforcement pluridisciplinaire que manifeste l'intérêt croisé des disciplines traditionnelles de l'art (critique d'art, histoire de l'art et esthétique) pour les questions spatiales - concepts et objets ${ }^{1}$ - et pour les textes de la géographie ${ }^{2}$, et inversement des géographies francophones ${ }^{3}$ et anglophones ${ }^{4}$ pour les pratiques, les objets et les notions issues de l'art ${ }^{5}$. Dans le cadre restreint de ce numéro spécial, cette tendance est confirmée par la représentation notable des jeunes chercheurs confirmés (postdoctorants) ou en thèse aussi bien dans la réponse à l'appel que dans la sélection ici présentée. Par le jeu de la cohabitation inédite de textes aux auteurs d'origine disciplinaire diverse (plasticiens, spécialistes d'esthétique, architectes, géographes) et d'un travail approfondi et collaboratif d'édition, ce numéro a l'ambition de proposer, des outils pour une transformation de l'intérêt en une expérience de co-construction d'outils théoriques et conceptuels pour une approche de la dimension spatiale de l'art contemporain - outils opératoires dans les différents champs disciplinaires concernés. Cette expérience est ici conduite soit autour d'études de cas mettant en évidence les données qui posent les conditions de possibilité de cette pluridisciplinarité (cf. les textes de C. Aventin, T. Gilabert, La Luna) ou fonctionnent comme des paradigmes (au sens strict) pour sa refondation théorique (cf. Ch. Ambrosino, C. Cartier, A. Lacan, J. Monier, C. Simond, A. Volvey et M. Houssay-Holzschuch), soit autour de mises au point/présentations bibliographiques lui servant de caisse de résonance, de prolongement et de complément (cf. Boutouyrie), soit enfin autour de notions ou de procédures fonctionnant comme autant de chevilles ouvrières de son articulation (cf. O. Bratosin, T. Castro, M. Gérardot, O. Melendez, M. Scarpulla, Ch. Ruby, H. Regnauld et Ch. Viart).

Mais il participe aussi de son renouvellement, puisque, comme en témoignent non seulement les propositions sélectionnées mais les réponses à l'appel prises dans leur ensemble, ce sont en grande partie de nouvelles formes d'expression artistique, de nouveaux objets et concepts, de nouvelles méthodes qui alimentent aujourd'hui cette pluridisciplinarité qui investit massivement l'art contemporain.

Tout d'abord, cette pluridisciplinarité se construit moins autour des formes accomplies et stabilisées dans des objets artistiques qu'autour des pratiques artistiques, tout particulièrement des manières de faire avec

\footnotetext{
${ }^{1}$ Voir à ce sujet Brayer, 1995 ; Tiberghien, 1995, 2007 ; Miles, 1997 ; DaCosta Kaufmann, 2004 ; Regimbald, 2008 ; Corbel, 2008. Le Colloque International organisé par le CIRHAC (Centre Inter-universitaire de Recherche en Histoire de l'Art), Université de Paris I, en mars 2004, intitulé "Atlas et les territoires du regard. Le géographique de l'histoire de l'art (XIXe et XXe siècles) » entrait dans l'art par les thèmes suivants: "création et lieux», "voyages, déplacements, exils réels et imaginaires ", "cartographies ", "le géographique aujourd'hui », «l'exposition comme image du monde » (Vanci-Perahim, 2006); la jeune revue en ligne Textimage a consacré en 2008 son numéro 2 aux «Cartes et Plans » et propose plusieurs articles sur la carte dans l'art et la littérature contemporains, dont celui de M. Regimbald (2008) intitulé "Quand l'art découvre ses cartes»; la collection Les Carnets du paysage (ENSP éd.) travaille depuis 1998 à une pluridisciplinarité (esthétique, architecture, géographie et paysagisme) autour de l'objet paysage.

${ }^{2}$ Voir, par exemple, Miles 1997 ; Kastner et Wallis, 1998 ; Tiberghien, 2001 ; Besse, 2000 ; DaCosta Kaufmann, 2004.

${ }^{3}$ Voir Grison, 2002, 2005 ; Staszak, 2006 ; Volvey, 2003 ; Giu, 2009 ; Gilabert, 2004 ; Boutouyrie, 2006 ; Blanc, 2009.

${ }^{4}$ Voir Chang et Lee, 2003 ; Chang, 2008 ; Minty, 2006 ; Pinder, 2005 ; Cant et Morris, 2006 ; Somdahl-Sands, 2006. A titre d'exemples de cette thématique émergente, le symposium au titre évocateur "Emerging Affinities? Art and Geography », organisé par J. Carter, G. Dunlop, M. Baker, F. McKee, K. Foster à l'université d'Edinbourg en mai 2006 ; la session «Art and geographical knowledge » de la RGS-IBG Annual Conference, Manchester 2009, autour du thème de l'art comme forme de connaissance géographique; la session "Geographies of art and the environment» de la conférence régionale de l'UGI, Glasgow 2004 (Cant et Morris, 2006); et les quatre sessions consacrées à "Geographies of Art» de la conférence annuelle de l'Association of American Geographers, Las Vegas 2009. La revue Cultural Geographies s'est fait l'écho en plusieurs occasions de ce nouvel intérêt pour l'art en accueillant des articles qui mobilisent les arts visuels (peinture et photographie) ou de la performance pour aborder avec un matériau et des références nouveaux, ses thèmes de prédilection, notamment le $n^{\circ} 9$ de 2002, $n^{\circ} 10$ de 2003 et le $n^{\circ} 13$ de 2006.

${ }^{5}$ Le symposium pluridisciplinaire "Activité artistique et spatialité », co-organisé par A. Boissière (philosophe), V. Fabbri (philosophe) et A. Volvey (géographe), qui s'est tenu à Lille-III, en mars 2007, et qui rassemblait des géographes, des philosophes spécialistes d'esthétique, des artistes et des psychanalystes, a posé les conditions d'une rencontre pour une pluridisciplinarité informée et dialogique.
} 
l'espace dans lesquelles l'art contemporain se trouve engagé depuis les années 1960. Dans leur grande majorité les textes rassemblés ici, qu'ils émanent des traditions disciplinaires liées à l'esthétique ou bien des cultures disciplinaires de l'espace, abordent l'art par les pratiques et placent l'objet d'art dans la perspective de celles-ci. Se donnant comme visée commune - conformément à l'orientation privilégiée par l'appel à propositions l'intelligibilité de la dimension spatiale de l'art, ils se sont majoritairement attachés à mettre en relief la dimension spatiale de l'activité artistique contemporaine, pour donner à comprendre la dimension proprement relationnelle des objets d'art contemporain aux lieux, leur qualité d'objets d'art-lieu (cf. ci-dessous). Cette perspective spatiale explique ce croisement disciplinaire inédit puisque les disciplines traditionnelles de l'art y rencontrent quelque peu leurs limites, pour des raisons de culture théorique ${ }^{6}$ aussi bien que méthodologique ${ }^{7}$, tandis que la géographie actorielle contemporaine y trouve un champ factuel nouveau et riche pour démontrer la pertinence et asseoir la visibilité de ses propositions théoriques et méthodologiques. Ce n'est pas, en effet, que l'art n'a jamais intéressé les géographes ${ }^{8}$, mais ils/elles trouvent aujourd'hui dans les pratiques artistiques contemporaines les raisons de faire rentrer cette activité dans le champ factuel de la géographie. Dans cette perspective, ce qui est proposé par les géographes impliqué/es dans ce numéro spécial relève moins, à mon sens, d'une géographie de l'art à proprement parler que d'une approche géographique de l'art, où d'une part, la géographie contemporaine (francophone, anglophone) fait preuve de sa fécondité à côté d'autres disciplines, et où d'autre part, elle s'ouvre à une pluridisciplinarité collaborative dont la transversalité et les circulations croisées lui permettent autant de renouveler des thématiques reconnues que d'en envisager de nouvelles, et où enfin elle trouve un appui pour participer au questionnement du régime de scientificité de la géographie contemporaine (cf. Regnauld et Viart). Inversement, cet élargissement du champ factuel de la géographie à l'art, montre l'importance de cette activité dans les formes contemporaines de re-création du collectif social (cf. La Luna, Aventin, Boutouyrie, Ruby).

B. Debarbieux (1995 : 880) a écrit «la littérature est sans doute la forme artistique dans laquelle l'imaginaire géographique d'un individu et d'une société trouve ses expressions les plus abouties » et R. Knafou (2003: 91) élargit cette position «l'art, représentation qui nous informe en profondeur sur le fonctionnement d'une société, participe puissamment à la construction des représentations de l'espace : il y a là, pour une géographie rompue désormais à l'étude des représentations spatiales, une source d'analyse importante et encore peu explorée, qu'il s'agisse de mieux 'lire' la ville ou de puiser dans les réserves sans fond d'une iconographie foisonnante »; la géographie sociale française après A. Frémont et G. Di Méo a sollicité les textes littéraires pour approcher l'espace vécu ou le territoire (on la trouve d'ailleurs représentée ici avec le texte de Bratosin). Pourtant c'est moins autour de l'ensemble 1- œuvre littéraire (cinématographique ou picturale) comme source ou fond iconographique, 2- description poétique comme opérateur et médiation de l'imaginaire/la représentation du (ou d'un) monde ou comme écriture géographique d'une image du monde qui lui préexisterait et qu'elle prélèverait sur le monde, 3- paysage comme forme stabilisée de la

\footnotetext{
${ }^{6}$ Le courant phénoménologique s'est particulièrement intéressé à la question de la spatialité, mais en faisant moins travailler la pratique que l'objet d'art (Heidegger, 1996) ou bien en rabattant cette question sur la logique corporelle (Straus, 1989; Maldiney, 1973), quand elle ne l'abordait de manière métaphorique. C'est pourquoi le symposium pluridisciplinaire lillois (cf. ci-dessus) proposait de réinvestir autrement ce cadre philosophique - précisément la phénoménologie (Maldiney, Straus), les psychanalyses existentielle (Binswanger) et transitionnelle (Winnicott, Anzieu) -, pour penser cette fois la dimension spatiale de l'activité artistique.

${ }^{7}$ L'esthétique, l'histoire de l'art, la critique d'art ne comptent ni le terrain ni l'archive administrative au nombre de leurs méthodologies disciplinaires gouvernées par le visuel et l'analyse de l'objet, comme le montre par exemple sa quête des æuvres du Land Art dans l'Ouest états-unien que relate G. Tiberghien (1996).

${ }^{8}$ Pour ce qui concerne les géographes français, par exemple les incursions dans la littérature d'A. Frémont, de G. Di Méo, de R. Brunet, mais aussi d'Y. Lacoste et les travaux sur les ceuvres littéraires de J.-L. Tissier ou de M. Brosseau (1996) sont connus, mais aussi les références au cinéma de M. Foucher ou aux arts plastiques d'H. Regnauld (1998) - voir aussi Hérodote (1987). Récemment les réflexions sur la peinture de A. Berque ont été menées pour fonder une définition culturaliste du paysage, l'approche postcoloniale de l'œuvre d'un Gauguin voyageur par J.-F. Staszak est venue étayer une réflexion sur l'exotisme, tandis que l'approche chorématique de la spatialité de l'art par L. Grison est venue augmenter le corpus de la géographie structurale. Ces derniers auteurs ont fait rentrer plus significativement l'art dans le champ factuel de la géographie.
} 
représentation/de l'imaginaire attaché/e au lieu, qu'autour d'un nouvel ensemble 1- art plastique/installation/danse/performance comme pratique/action participative et observable, 2- terrain (fieldworking) et cartographie (mapping) comme fabriques spatiales d'une signification attachée aux lieux et aux moments de la pratique, 3- carte comme médiation, opérateur et construit de ce sens, que fonctionne la pluridisciplinarité ici (bien) représentée. L'importance du cartographique, comme alternative au paysage, dans les arts contemporains ${ }^{9}$ est reconnue par les spécialistes d'esthétique (Tiberghien, 1995, 2007; VanciPerahim, 2006 ; Textimage, 2008) et en particulier par la philosophe M.-A. Brayer qui en a fait un sujet d'exposition (2000) aussi bien qu'un objet d'étude (Brayer, 1995). Teresa Castro s'en fait elle aussi l'écho dans ce numéro et l'instaure en indice du "tournant spatial" des arts contemporains et des disciplines qui les étudient (voir aussi Castro, 2008). Cependant ce centrage nouveau sur l'écriture cartographique et sur la carte articule cette pluridisciplinarité sur une ambiguïté qu'il est indispensable de lever afin qu'elle ne devienne ni biais ni pierre d'achoppement : pour les disciplines de l'art, le géographique est (n'est) reconnu (que) sous les traits du cartographique, cette «géo-graphie » apparaissant comme la justification de la rencontre avec la science géographique contemporaine ${ }^{10}$. Dans ces conditions la pluridisciplinarité est appelée à décevoir les uns - ceux qui n'auront pas retrouvé leur objet en géographie -, et les autres - ceux qui ne se sentiront pas leur construit disciplinaire reconnu par les premiers -, à rater son objet : la géographie n'est pas, n'est plus science cartographique, mais bien, en tant que science de la dimension spatiale du social, une grande productrice et consommatrice de ces artéfacts qui mobilisent une écriture spatiale, et partant, une connaisseuse éclairée, voire une théoricienne, de la cartographie. Abandonnant une définition exclusivement informationnelle de la carte (supposant un rapport d'analogie entre la carte - l'énoncé, et le monde - son référent), la géographie a proposé une définition textualiste inscrite dans la crise générale de la représentation (un énoncé autoréférentiel) et une définition praxéologique (Mondada, 2000) où la carte est à la fois objet du discours dans les pratiques de négociation et outil performatif et configurant de transformation du monde. Ce choix de définitions la rend apte à comprendre les usages non (uniquement) représentationnels de la carte/cartographie qui ont cours dans l'art contemporain.

La problématique centrale de cette pluridisciplinarité renouvelée autour de pratiques artistiques qui articulent objet et lieu à travers des actions, est politique (voir Van Essche, 2007). Pour autant qu'il est entendu que l'art contemporain ne fait pas du politique avec des images politiques, selon une politique de la représentation, mais que, comme le formule joliment l'artiste français Ernest Pignon-Ernest, «c'est la manière de faire de l'art qui est politique » (in Briot et Humblot, 1980 : 32), selon une politique de l'action (cf. Volvey et Houssay-Hozschuch, La Luna, Ruby, Aventin, Boutouyrie). Ce qui est en jeu ici, c'est la capacité de l'art contemporain comme pratique (ou ensemble de pratiques) à créer des situations qui articulent une spatialité et une temporalité données autour d'un projet individuel ou collectif par le truchement de procédures interactionnelles et contractuelles, situations qui créent les conditions de possibilité du dégagement de significations attachées aux lieux (cf. Ambrosino, Cartier, La Luna) et de leur mise en jeu, en forme et en vue dans des objets d'art-lieux (cf. Scarpulla, Gilabert, Volvey et Houssay-Holzschuch). Cet art, qui s'insère et/ou

\footnotetext{
${ }^{9}$ Objet qu'on trouve aussi dans l'art musical contemporain chez. Annea Lockwood par exemple, qui collecte des sons de volcans, de tremblements de terre, de fleuves, etc., et les élabore en "cartographies sonores" au travers d'installations associant sons, cartes, images et entretiens (A sound map of the Danube, 2008). Je remercie C. Giu pour cette information concernant la production musicale contemporaine.

${ }^{10}$ Elle concerne même la littérature contemporaine qui croit rencontrer la géographie en découvrant les cartes ou les croquis de lieu : "Une fois la zone blanche localisée, j'essayais de décrire le plus précisément que je le pouvais la configuration des lieux. Je prenais ma mission très au sérieux et m'étais muni de tous les outils outils de l'exploration traditionnelle : une balise GPS, un appareil photo, ainsi qu'un carnet de croquis sur lequel je prenais des notes, effectuais des relevés et dessinais des plans sommaires. A accumuler ainsi des informations et à me glisser par-dessus les murs et les palissades, j'avais l'impression de faire de la géographie parallèle, alternative, à rebours de la science officielle, forcément impersonnelle et réductrice. Deux rencontres sont venues corriger ce sentiment : celle, d'abord, d'un vrai géographe, qui faisait au quotidien ce que je prétendais faire en amateur, à savoir mettre à jour les cartes. » (Vasset, 2007 : 35 36). Voir aussi dans Textimage (2008), les articles sur Le Clezio et Hocquard.
} 
s'implique dans la cité déploie son action dans un horizon de temps limité (art processuel et producteur d'objets le plus souvent éphémères : il est présentiste), pour lever des questions/conflits qui dépassent le strict corpus esthétique et faire de celles/ceux-ci les leviers de significations en révélant et en faisant fonctionner des mécanismes internes à ce dispositif social, atteint ce faisant une dimension globale (tous les domaines de la vie sociale sont touchés et constituent à la fois le contexte et les ressources de l'action). Sa manière de participer à l'invention d'un vivre-ensemble situé (i.e. à la fois localisé et intrinsèquement lié à l'ensemble de situations constituant son contexte d'émergence et de finalisation), sa manière de reconstruire du lien ou des médiations absentées en faisant émerger des significations et en les construisant en micro récits partagés, sa capacité à transformer les communautés territorialisées (politiques, administrateurs, résidents, usagers, etc.) en maîtres d'ouvrage de leur destin collectif et/ou de leur identité collective, en font un maitre d'œuvre plus recherché et aussi plus instrumentalisé que l'architecte par les responsables de l'aménagement ou du marketing territorial. Sa pratique fait de l'artiste aujourd'hui un nouveau venu dans l'ensemble des sciences de l'espace (cf. Regnauld et Viart, Aventin, La Luna, Boutouyrie). Quelles que soient les formes d'expression artistique impliquées aujourd'hui dans cette entreprise politique et quels que soient les objets concrétisés (ou pas) avec lesquels elle s'offre à l'expérience esthétique, cette manière de faire travailler le(s) lieu(x) avec l'art et de faire travailler l'art avec les lieux (entendus dans leur triple dimension matérielle, idéelle et virtuelle) instaure une catégorie transversale à l'art contemporain et à ses découpages classiques par type d'objets ou de média. Je propose d'appeler ces pratiques Land Arts parce qu'à mon sens, elles sont de diverses manières des héritières du Land Art états-unien dans ce qui en a fait à la fois son unité et sa radicalité, et qui n'a jamais été théorisé : son rapport au land - rapport construit dans la pratique -, sa manière fondamentale de travailler la question de l'usage du sol (Volvey, 2003, 2005).

\title{
Définir le Land Art à partir du land : vers une intelligibilité des spatialités de l'art contemporain
}

\author{
Le travail du land : land claiming (revendiquer le sol) / land working (oeuvrer \\ le lieu)
}

Ainsi, j'aimerais à la faveur de cette introduction préciser ce moment paradigmatique insoupçonné du Land Art états-unien et, ce faisant, créer peut-être les conditions conceptuelles et théoriques d'une compréhension renouvelée des pratiques artistiques contemporaines, et proposer quelques éléments pour une lecture transversale des textes rassemblés ici. Appuyée sur une lecture pluridisciplinaire du Land Art, cette proposition développe un point de vue géographique sur l'art contemporain.

Le Land Art états-unien est reconnu pour une constellation d'artistes (Tiberghien, 1995) qui ont travaillé dans la seconde moitié des années 1960 et dans les années 1970 dans des directions similaires de sorte que, croisant sans cesse leurs trajectoires respectives, ils ont exposé ensemble aux Etats-Unis et en Europe ${ }^{11}$, ont mis en vue des objets d'art formellement comparables créés au moyen de démarches elles-mêmes comparables et rapportés à des référents culturels partagés (mythologiques, cosmologiques). La critique d'art, l'histoire de l'art et l'esthétique associent traditionnellement aux objets d'art du Land Art pour les définir les termes d'in situ et d'outdoors (cf. Volvey et Houssay-Holzschuch). Ces deux termes contiennent, en effet, les éléments de la radicalité de cette pratique artistique qui, à côté et dans le prolongement d'autres propositions mais à sa manière, a mis en cause d'une part, le rapport de représentation entre l'objet d'art et la réalité, d'autre

\footnotetext{
${ }^{11}$ Par exemple : Earthworks, 1968, Dwan Gallery, New York ; Earth Art, 1969, Andrew Dickson White Museum, Ithaca, NY ; Earthworks : Land Reclamation as Sculpture, 1979, King County, Washington; et, en Europe, When attitudes become form, 1969, Bern, Suisse ; Sonsbeek 71 Buiten de perken, 1971, Sonsbeek Park, Arnhem, Pays-Bas.
} 
part, le rapport conventionnel, dissymétrique et mercantile entre les acteurs du «monde de l'art (Becker, 1988) institué. Le Land Art s'est employé à disqualifier la dimension représentationnelle des objets d'art en même temps que la prétention des institutions muséales à être les lieux centraux de l'art (Penders, 2000). C'est une pratique artistique qui s'effectue «hors les murs de l'art»-hors du lieu physique, musée et galerie, et par là-même en dehors des relations instituées et codées qui lui sont attachées -, pour recomposer autour du projet d'art un autre «monde de l'art » situé $^{12}$ - un ensemble de relations relatives et situées, attachées au processus de l'œuvre en train de se faire et à sa mise en vue. Outdoors ne signifie pas, en effet, «en plein air » mais désigne le modus operandi d'une pratique qui se fait contre l'institution muséale ou le système de relations formant le monde de l'art et dont l'institution muséale constitue le centre ${ }^{13}$. Une pratique moins centrée sur la production d'objets entretenant un rapport de représentation avec des objets extérieurs que sur la découverte et le traitement d'une réalité extérieure au monde de l'art, qui sera construite dans une idée d'objet, élaborée et agit dans un projet situé et, éventuellement, mise en vue dans un objet d'art sur le lieu de son travail. Comme l'énonçait Dennis Oppenheim : "l'objet était vraiment la cible. L'objet d'art était ce à quoi l'on tentait de trouver une alternative » et, ce faisant, «le lieu prit en quelque sorte la place de l'objet. (...) La nécessité de reproduire, de copier, ou de manipuler la forme n'était plus une fin en soi. » (in Tiberghien, $1995: 98)^{14}$. L'objet d'art était une cible à travers laquelle l'ensemble du système de l'art incriminé pouvait être atteint et, inversement, le travail de l'art avec le lieu une solution pour en sortir ${ }^{15}$. Avec sa "dématérialisation » d'une part, disparaît le support de l'encodage de l'art par le monde de l'art institué et le véhicule de la relation dissymétrique entre ses acteurs, le produit du rapport de représentation à la réalité, le moyen de la marchandisation de l'art, et, avec la délocalisation outdoors de l'activité artistique et de l'expérience esthétique d'autre part, le lieu devient la ressource de l'art et sa mise en œuvre artistique la condition de la recomposition d'un monde de l'art situé. C'est pourquoi la radicalité du Land Art ne réside pas dans un simple changement de matériau pour le façonnage d'objets ou de référent pour la représentation de sujets - ce que laissait entendre le vocable Earthwork (en tant que travail de la terre ou Terre), première dénomination du Land Art -, mais bien dans sa spatialité ou sa manière de faire avec l'espace ${ }^{16}$.

"Because I wasn't very excited about objects wich protude from the ground. I felt this implied an embellishment of external space. To me a piece of sculpture inside a room is a disruption of interior space. It's a protusion, an unnecessary addition to what could be a sufficient space in itself. " Oppenheim (in Kastner et Wallis, 1998 : 202).

\footnotetext{
${ }^{12}$ Pour H. Becker (1988) : "Un monde de l'art se compose de toutes les personnes dont les activités sont nécessaires à la production des œuvres particulières que ce monde-là (et d'autres éventuellement) définit comme de l'art. »

${ }^{13}$ L'artiste états-unien Christo résume dans son entretien avec A.-F. Penders (1995:25) cette position commune des land artists et laisse deviner ses conséquences: "A.-F. P. : Vous dites que tout est esthétique. En 1961, quand vous avez décidé de travailler à l'extérieur, était-ce un rejet du système des galeries ? (...) Christo : Avec ce projet, je voulais bousculer notre notion de l'art. Sortir du Musée, hors de la galerie, loin de ce qui pourrait être appelé l'espace contrôlé de l'art. Bien sûr ces projets prenaient possession d'un espace public. Ils étaient conçus pour questionner la notion habituelle de l'art dans une galerie, un musée ou un espace "protégé", un espace régit par des règles. (...) Nos projets, en allant au-delà de ces lieux, devenaient autre chose. Une symbiose entre l'art, l'architecture et l'urbanisme. »

${ }^{14}$ En écho ces citations de R. Smithson/ «I began to question very seriously the whole notion of Gestalt, the thing itself, specific objects. (...) I had to question where the works where, what they were about. So it became a preoccupation with place » (in Kastner et Wallis, 1998 : 30-31) ; " The more compelling artists today are concerned with 'place' or 'site'. » (ibid. p. 25).

${ }^{15}$ Cette position est objectivée dans de nombreuses ceuvres de land artists. Que ce soit la série des Nonsite, 1968 de R. Smithson qui expose dans un musée les matériaux (substrat rocheux ou terrassement) prélevés dans un port ou un aéroport dans des compartiments minimalistes évoquant des containeurs, et les indexe sur les lieux de leur collecte par le truchement d'une documentation cartographique et photographique. Que ce soit le long travail artistique de Christo avec l'empaquetage -empaquetage d'objets d'art (tableaux de paysage ou portraits), empaquetage des sujets de la représentation (corps nus et pays), en passant par empaquetage de vitrines et de musées (lieux d'exposition/vente des objets d'art) - pour disqualifier la dimension représentationnelle de l'art et les institutions muséales, pour transporter l'activité artistique et l'activité esthétique hors les murs de l'art.

${ }^{16}$ Celle-ci est très clairement énoncée dans le texte d'introduction " From Exhibition to Activity » de l'exposition Sonsbeek 71, Buiten de perken, Arnhem, juin-août 1971, à laquelle ont participé plusieurs land artists états-uniens (Beern, 1971).
} 
D. Oppenheim est sans doute le land artist qui objective le mieux cette nouvelle problématique spatiale. Ainsi, dans sa série de 1969 Gallery Transplant, Oppenheim expose le montage d'une photographie donnant à voir une performance qu'il a réalisée dans le Bird Sanctuary d'Ithaca (New York) et à Kearny (New Jersey) - l'artiste traçant au sol et hors les murs le plan grandeur nature d'une salle d'un des musées dans lequel il a été invité à exposer -, d'une carte topographique sur laquelle il localise la performance, du plan du musée sur lequel la salle concernée est délinéée. D'un point de vue spatial qu'a-t-il fait ? : 1- il a disloqué l'espace muséal en sélectionnant et en détachant une salle de l'ensemble des salles qui constitue le musée, 2- il a délocalisé cette salle en lui donnant d'autres coordonnées géographiques et en changeant sa place relative dans l'espace relationnel habituel de l'art (elle est alors placée en dehors et à l'écart du monde de l'art institué, et en périphérie urbaine), 3- il l'a re-localisée dans un geste graphique et, 4- ce faisant, il a activé en elle une autre fonction - la création d'objet d'art-, que celle qui lui est traditionnellement dévolue - l'exposition d'objet - : une œuvre d'art a lieu à l'intérieur de cette aire délimitée, unifiée et qualifiée par ce tracé au sol, par simple activation de ses propriétés locales (Oppenheim parle d'«activated surface»). Qu'est-ce que cet objet d'art ? Il ne s'agit plus d'art dans un lieu : soit un objet exposé quelque part et qui entretient avec le lieu où il se tient un rapport d'extériorité, celui-ci lui servant de réceptacle ou de condition de mise en vue ${ }^{17}$. L'objet d'art ne se trouve pas seulement dans un lieu (ou une localité), il est $d u$ lieu et surtout avec le lieu, il est objetlieu.

Pour aborder cette dimension intrinsèquement spatiale de l'objet d'art, il faut abandonner une conception essentiellement positionnelle du rapport entre les choses et les lieux qu'elles occupent (Tiberghien, 1995), soit une conception centrée sur les objets fabriqués par les artistes, pour promouvoir une conception relationnelle des lieux aux choses qui les occupent (Volvey, 2003, 2005) ${ }^{18}$. Cette conception, pour laquelle je propose de recourir à la distinction entre le lieu comme topos et le lieu comme chôra que théorise le géographe français A. Berque (2003), met l'accent sur le mouvement de liaison chose/lieu, et partant, sur ce qui les lie : l'action artistique - qui met en lieu (et non pas seulement localise) l'art et « œuvre d'art » le lieu ${ }^{19}$. On mesure ainsi les limites conceptuelles de la notion d'in situ rapportée au Land Art états-unien: faisant valoir un rapport d'extériorité entre le lieu (topos) et l'objet marqué par "in", elle ne donne pas à comprendre le rapport de liaison dynamique et de concrétisation réciproque de l'objet et du lieu qui se noue dans la pratique artistique. En somme, il ne suffit pas de reconnaître la filiation du Land Art, via l'art minimaliste, avec la statuaire de Brancusi ou les problèmes d'installation du Balzac de Rodin (Krauss, 1979; Tiberghien, 1995), mais de voir comment avec le Land Art la problématique de l'abolition du socle de l'objet d'art trouve son aboutissement, via la dématérialisation de l'objet et la disqualification de l'institution muséale, dans le rapport de liaison dynamique de l'art et du lieu. Le lieu n'est pas un nouveau socle pour un objet d'art in situ, mais l'élément dynamique d'une chôra. Une telle définition chôraïque de l'objet d'art invite à réfléchir sur le Land Art en adoptant un point de vue chôrésique (spatial, génétique et relationnel) centré sur les fabriques spatiales de l'objet. Cette perspective permet de retrouver le land dans le Land Art, de le faire travailler, de le recharger sémantiquement pour proposer une définition de ce mouvement artistique qui prenne pleinement en compte sa dimension spatiale, qui en fasse l'origine de son unité et de sa radicalité et, partant, la matrice d'une intelligibilité général des Land Arts contemporains (cf. Volvey et Houssay-Holzschuch).

\footnotetext{
${ }^{17}$ «Il s'agit ainsi d'un lieu et d'espace et non pas d'objets dans un lieu. » disait R. Morris à propos de son Observatory, Velsen, 1971 pour Sonsbeek 71, Buiten de Perken (in Tiberghien, 1995 : 102).

${ }^{18}$ Une autre manière, plus paradigmatique (au sens strict), de le dire proposerait de considérer le Land Art moins dans la perspective des objets d'art de Michael Heizer et des textes de Robert Smithson, et plus dans celle des œuvres et discours autorisés de Dennis Oppenheim et de Christo et Jeanne-Claude.

${ }^{19}$ Il est tout à fait inadéquat de recourir ici la notion d' " artialisation » créée par le philosophe A. Roger (1978). Celle-ci désigne en effet les deux manières (de visu et in situ) dont l'art est projeté dans la nature ou sur le corps transformant le pays en paysage et la nudité en $n u$, et relève explicitement des logiques conjointes de la représentation artistique et de la contemplation esthétique en tant que schème culturel organisant l'une et l'autre opération. L'expression et jeu de mots « œuvrer d'art » (Les Cahiers d'EspaceTemps, 2002) me paraît plus à même de rendre compte de ce qui est en jeu ici: l'action artistique de transformation du monde (cf. Volvey et HoussayHolzschuch).
} 
«Recently I have been taking galleries apart, slowly. I have a proposal that involves removing the floorboards and eventually taking the entire floor out. I feel this is a creeping back to home site. » Oppenheim (in Kastner et Wallis, 1998 : 203).

Pour comprendre comment le land est impliqué dans cette chôrésie, il convient de rappeler le contexte juridico-culturel et historique du Land Art et d'articuler entre elles les problématiques du lieu et de l'outdoors. Car si «le lieu a bien pris la place de l'objet» (Oppenheim), la stratégie d'outdoors a été dans le contexte foncier états-unien la condition de la mise en œuvre de ce programme. En effet, le déplacement hors les murs de l'activité artistique, implique que l'artiste puisse faire un usage artistique d'un terrain qui n'a pas été affecté de manière coutumière ou réglementaire à cet usage, qui est approprié par une personne privée ou une institution, et dont la mobilisation est encadrée par une réglementation territoriale ou sectorielle : l'artiste ne dispose plus de lieux institués - le musée/la galerie, l'atelier -, à la fonction artistique prédéterminée, il inscrit sa pratique sur la Frontier de l'art. La réalisation outdoors d'un objet d'art-lieu suppose outre la sélection d'un lieu, son jalonnement et l'obtention d'une autorisation d'en faire un usage artistique temporaire ou définitif. Elle impose par conséquent que l'artiste conduise une procédure de revendication (claiming) d'un terrain (land) pour en faire usage artistique. Or dans le contexte juridique états-unien la procédure de land claiming réglée par le Homestead Act de 1862 fait dépendre le droit d'accès à la terre de son travail (de Soto, 2005 ) et se déroule de la manière suivante : 1- jalonnement d'un terrain («to stake out a claim» ou «to locate a claim »), 2- travail de la parcelle jalonnée sur une temporalité donnée (5 ans ou 6 mois dans le cas d'un achat de la parcelle), 3- reconnaissance légale des droits d'usage et de jouissance de la parcelle, sanctionnée par un titre de propriété. Dans ce contexte juridico-culturel, land claiming et land working vont de paire. La réalisation outdoors d'un projet d'objet d'art-lieu implique un land claiming dont l'aboutissement dépend d'un land working : le land est à la fois terrain (track of land) pour un usage artistique, champ d'un travail artistique (fieldwork), concrétisation de l'idée d'objet et du lieu dans un objet d'art-lieu (chôra).

L'enjeu foncier (propriété et usage du sol) est au cœur du Land Art états-unien, comme l'objective D. Oppenheim à la fois dans Gallery Transplant où le tracé au sol de la galerie évoque le découpage du sol étatsunien en townships, le traçage l'opération de jalonnement et le pas de l'artiste l'opération d'arpentage, et dans une série antérieure Site Marker. Si le land claiming n'est dans cette œuvre de 1967 que photographique, Oppenheim mobilise explicitement le registre foncier pour la commenter, annonçant le travail ultérieur avec le lieu qu'il accomplira pour Gallery Transplant [c'est moi qui souligne] : «In my site markers of 1967 the notion of travel was coupled with the sense of place. Place kind of took place of the object... My simple act of issuing a stake and taking up a photograph of the piece and claiming, pointing out where it was on a map and describing it on the document was sufficient... The need to replicate, duplicate or manipulate form was no longer an issue. » (in Kastner et Wallis, 1998 : 30). Pour sa part, le couple de land artists états-uniens Christo et Jeanne-Claude résume cette nouvelle perspective en proclamant justement «L'essentiel de [nos] projets est la prise de possession de l'espace » (in Yanagi, 1989 : 198). Cet enjeu inscrit pleinement le Land Art étatsunien dans la culture foncière états-unienne et plus contextuellement dans le mouvement de la contre-culture de la fin des années 1960, dont l'un des chevaux de bataille a été la mise en cause du rapport prédateur et destructeur de la société états-unienne à la terre (sols et ressources). Cette contre-culture s'est exprimée en particulier à travers le mouvement civique du Land Use Reform qui concentra son action sur la question du contrôle de l'aménagement et de l'usage des sols et ressources. Ce mouvement déboucha sur le National Environmental Policy Act de 1970 - loi cadre votée par le Congrès qui imposait à l'Etat fédéral une responsabilité en matière de maintien des équilibres entre les ressources naturelles et leur utilisation à l'échelle des Etats-Unis -, sur l'abrogation du Homestead Act sur les terres fédérales (Federal Land Policy and Management Act, 1976) et sur la mise en place des institutions et des programmes du Land Reclamation ${ }^{20}$

${ }^{20}$ Processus de réhabilitation des terrains et ressources détériorés (sol, végétation, eau) par une activité humaine afin de rétablir des conditions équivalentes à celles qui prévalaient avant la détérioration, soit dans la perspective d'un nouvel usage (reconversion) soit dans celle d'un classement en réserve naturelle (restauration et conversion). 
(1973 et 1977). L'association land claiming/land working imposée par la stratégie d'outdoors du Land Art états-unien $^{21}$ est au principe même de la chôrésie : en elle, un monde de l'art évolutif et relatif à l'œuvre en train de se faire se recompose au travers de situations instaurées par l'artiste ${ }^{22}$, et par elle, la chôra gagne en significations pour atteindre sa monumentalité.

\section{Lever des significations : les fabriques spatiales du Land Art}

«At the moment a painter is mixing paint, he is a chemist. If it is too thick, he must add turpentine. I am the same, except that my chemistry is made of landowners, politicians, engineers, workers, attorneys, fabric, steel, mountains, tees, roads, houses, bridges, rivers, winds and light. » (Christo in Los Angeles Times, 1991).

La concrétisation de l'idée d'objet et du lieu (chôra) dépend singulièrement de la méthode artistique, soit l'ensemble des procédures à travers lesquelles l'action artistique est conduite dans le cadre de la revendication et du travail du land (land claiming/land working). Ces fabriques de l'œuvre sont de deux types : le terrain (fieldwork) et le cartographique (mapping). Situations pluriactorielles créées par/avec l'action artistique, elles sont ce par quoi se réalise la liaison dynamique de l'idée d'objet et du lieu : elles sont les instruments de la mise en lieu de l'activité artistique et de la mise en œuvre artistique du lieu, de la concrétisation choraïque de l'art et du lieu. Ce sont des fabriques spatiales, au sens d'abord où elles font avec l'espace (et non pas dans l'espace) - l'espace dans ses dimensions matérielles et idéelles y est à la fois condition de la pratique et ressource de l'œuvre -, au sens où elles mobilisent des pratiques à forte dimension spatiale, et au sens enfin où elles spatialisent cette ressource pour la construire dans un objet d'art-lieu l'espace est un construit de ces situations artistiques. À travers elles, la chorésie ordonne moins une rematérialisation de l'objet que sa re-sémantisation.

Le terrain artistique implique deux grands domaines de la vie des sociétés : une manière politique et une manière scientifique de (faire) travailler le land. S'agissant du terrain scientifique (observations, mesures, enquêtes, entretiens, expérimentations en milieu ouvert), il s'organise en actes de recherche à visée cognitive impliquant à la fois les sciences empiriques fondamentales et les sciences de l'ingénierie. Il est le plus souvent orienté vers un savoir d'action - i.e. visant la transformation du lieu - et il est commandé par une logique de maîtrise des situations, il donne plutôt lieu à un encyclopédisme du factuel à la fois diagnostique et prédictif. Il instaure un monde de l'art pluri-actoriel peuplé de scientifiques, d'experts et d'ingénieurs. Mais sa visée cognitive peut être plus fondamentale, quand il fait émerger une intelligibilité proprement géographique du

\footnotetext{
${ }^{21}$ Il est temps de préciser ce qualificatif «états-unien » employé ici pour le distinguer du Land Art européen de la même époque. L'opposition entre le Land Art étasunien et le Land Art européen a été construite autour de l'objet d'art - c'est la question de sa taille -, et de la forme d'intervention dans/avec le réel de l'artiste - c'est la question du grand chantier. Elle est reconnue et entérinée par les critiques, et a donné lieu à des confrontations entre les artistes de l'un et l'autre continent portant sur la question du respect de la nature. Les land artists états-uniens sont réputés travailler au bulldozer des œuvres monumentales : R. Long qualifie R. Smithson d'Urban Cow-boy et R. Serra de «Rambo de l'art» (in C. Garraud, 1994 : 46). On voit qu'une autre manière de les distinguer mettrait en avant la question du land pour dépasser une fois encore une compréhension physicaliste du land dans une conception juridico-politique. La stratégie d'outdoors du Land Art états-unien, du fait de son contexte politique (une certaine culture du rapport au sol devenu sujet de controverses à la fin des années 1960), met moins la terre en chantier (Earth Work) pour produire des sculptures monumentales qu'elle ne prend possession du sol pour l'œuvrer d'art (Land Art).

${ }^{22}$ Nous retrouvons le complément de définition du monde de l'art donné par H. Becker (1988: 22) : « réseau de tous ceux dont les activités coordonnées grâce à une connaissance commune des moyens conventionnels de travail, concourent à la production des auvres qui font précisément la notoriété du monde de l'art», qui met l'accent sur le réseau de relations relatif au processus artistique et qui conditionne la légitimité artistique à des règles et des procédures organisées en schème de coopération, partagé par les acteurs de ce monde.
} 
lieu, un savoir spatial qui est construit dans l'objet d'art-lieu ${ }^{23}$. Le terrain politique, quant à lui, comprend toutes les formes d'interlocution et de négociation (lobbying, porte-à-porte, audience publique, expositions documentaires, usage des média, etc.) que le land artist doit mener pour obtenir l'autorisation de faire un usage artistique d'un sol. Il instaure un monde de l'art pluri-actoriel fait de juristes, de politiques, d'administrateurs, d'usagers et résidents, de journalistes, de directeurs de musées/galeries et de commissaires d'exposition. Ce terrain donne lieu à au moins deux types de savoir. D'une part, un savoir juridico-politique de type procédural (savoir relatif aux règles du fonctionnement politique d'un collectif social donné, aux régimes de propriété et à l'exercice des compétences territoriales et sectorielles, aux réglementations territoriales et sectorielles, etc.), d'autre part, un savoir de type idéel ou symbolique, dont la spécificité est qu'il émerge du et se co-construit dans le terrain comme un savoir $d u$ lieu pour le collectif d'acteurs. Le terrain politique est proprement symboligène, les procédures qui lui sont liées permettent de trouver/créer et d'objectiver la dimension idéelle du land et de la construire comme ressource dans l'objet d'art. L'ensemble de ces savoirs issus des situations scientifiques ou politiques est construit dans l'objet d'art-lieu. Les deux terrains ne sont pas hermétiques l'un à l'autre : pratiques conjointes, échange et réutilisation de données dans le cadre des procédures politico-administratives (l'encyclopédisme du factuel fait la matière des rapports d'impact environnementaux ou des rapports d'impact sociaux qui sont demandés aux land artists dans la procédure de land claiming) articulent et mettent en résonance les construits matériels et idéels du lieu. Le monde de l'art instauré par la revendication et le travail du land dans la pratique de terrain relève de domaines de la société extrêmement nombreux et variés : le Land Art est un art global.

"It [Earth Art] was radical, if you consider late 1967 or 1968 as the time when most of these huge pieces were done. I defend the approach of radicality, the fact that outdoor works invited dialogue with real time in ways art had not done before. » (Oppenheim, in Kastner et Wallis, 1998 : 226).

C'est parce que le land artist projette de faire un usage artistique d'un sol non affecté à l'art et doit le travailler pour en obtenir l'autorisation, qu'il mobilise le collectif social et peut l'œuvrer d'art via des pratiques situées et transactionnelles propres à faire «lever» les ressources matérielles et idéelles des lieux pour les construire dans l'objet d'art-lieu. En remplaçant l'objet par le lieu et le monde de l'art institué par un monde de l'art situé, le Land Art abandonne l'autodéfinition de l'art (trouver une signification en lui-même) - celle de l'art minimaliste, par exemple -, pour trouver/créer une signification attachée au lieu et co-construite dans l'action artistique via les pratiques de terrain pluri-actorielles et pluridisciplinaires. En l'abordant par le foncier, le projet land artistique travaille le collectif social radicalement et fondamentalement, le conduit à coconstruire un monde qui n'est pas insensé pour lui, un monde tissé de significations situées - i.e. relatives aux situations qui les a faites émerger et qui les a élaborées -, fixées dans des objets concrets qui disent le sens présent (ou la fiction de sens), et par conséquent, le plus souvent, éphémères. Cette construction de significations par un collectif social via la co-action artistique s'apparente ainsi à d'autres régimes de construction de fictions collectives en micro récits, le développement territorial (un récit qu'un collectif coconstruit comme motif présentiste d'un vivre-ensemble) : de là son instrumentalisation possible dans le marketing territorial pour la construction de la figure du lieu ou de l'édile ${ }^{24}$.

\footnotetext{
${ }^{23}$ Ce savoir géographique et sa construction dans l'objet d'art est énoncé dans cette citation de Christo à propos de Running Fence : «For a long time I was interested in doing a project in California. (...) Perhaps this is the most American State in the way of living. You know, the people live horizontally, in a very complex relation between urban, suburban, and rural life, and of course through all that sort of living they have a huge, acute notion about the land, the use of the land, driving to the land. And now they have the most highly advanced laws restricting land use and working on the land, and this is why the project should be rooted in a community with a fantastically acute and very nervous relation to the land. (...) This is why the project was horizontal, and the 24,5 miles are dealing with these urban, surburban and rural situations. » (in Diamonstein, 1979: 86).

${ }^{24}$ H. Becker toujours $(1988: 110)$ : «dans un monde de l'art, toute fonction peut être tenue pour artistique, et tout ce que fait un artiste, même le plus incontesté, peut devenir une activité de renfort pour quelqu'un d'autre. »
} 
Les terrains de l'action artistique confèrent des dimensions spatiales floues et complexes à l'œuvre d'art. Certains champs d'opération ont pour dimension le lieu revendiqué pour un usage artistique et le recouvrent presque totalement pour l'œuvrer de manière quasi exhaustive (terrain scientifique), d'autres une dimension non commensurable et une configuration non congruente à celui-ci (terrain politique). C'est pourtant bien le land qui est œuvré à partir des lieux d'interlocution (centres administratifs, par exemple) où la possibilité de son usage artistique est discutée, planifiée et mise en forme dans un cahier des charges. Quels que soient leur métrique et leur ordre de grandeur, les opérations de terrain participent, en tous les lieux qu'elles instaurent en contextes d'action, au levé des ressources matérielles et idéelles du lieu qui sont construites dans l'objet d'art-lieu. Ainsi les dimensions spatiales du land et de l'œuvre d'art (l'ensemble des lieux œuvrés avec l'action artistique) ne sauraient être confondues, bien qu'elles dépendent et s'informent l'une l'autre, et construisent dans leur interspatialité la spatialité relative et relationnelle de l'objet d'art-lieu. Mais c'est le propre même d'une conception choraïque du lieu que cette incommensurabilité du topos et de la chôra (Berque, 2003), par laquelle la/les signification/s advienne/nt.

Le Land Art n'est pas le seul mouvement artistique contemporain associé à la redécouverte de la carte et de la cartographie (Brayer, 1995 ; Tiberghien, 2007 ; Textimage, 2008), mais on conçoit que ces dernières sont étroitement liées aux stratégies propres au Land Art (Volvey, 2003). L'importance de la carte est reconnue pour l'ensemble des land artists états-uniens (Tiberghien, 1995) et particulièrement pour Robert Smithson (Corbel, 2008), Christo et Jeanne-Claude (Volvey, 2002, 2003, 2005). En effet, la stratégie foncière des land artists, principe de leur stratégie artistique et esthétique, s'articule à plusieurs niveaux à l'outil de spatialisation qu'est la cartographie et à son construit artéfactuel, la carte ou l'aerial map ${ }^{25}$. Tout d'abord et très simplement, la carte est l'outil de l'outdoors : s'orienter, explorer, établir les coordonnées du lieu qui « prend la place de l'objet ». C'est à ce titre que les quadrangle maps sont montées dans la documentation des Gallery Transplant, 1969 (Oppenheim) et de nombre de réalisations du Land Art. Tandis que le plan (cadastral) est le construit normal des actions foncières (land claiming). La carte est surtout un moyen pour sortir de la perspective linéaire - la forme classique de la spatialisation du monde pour sa représentation dans le tableau de paysage et son expérience esthétique. Elle lui substitue une autre forme symbolique pour conduire aussi bien la spatialisation de l'objet d'art-lieu que son expérience esthétique, tout en jouant de son effet de réalisme. La série des Gallery Transplant, 1969 de D. Oppenheim objective là encore ce passage du paysage à la carte, en faisant de la cartographie l'opérateur des transformations artistiques et esthétiques appelées par le Land Art : il instaure par un tracé au sol (écriture) un dispositif visuel vertical (point de vue zénithal) dans lequel le découpage du sol (township) vient se mouler dans le quadrillage de la projection cartographique (longitude/latitude) pour délinéer un champ d'action possible pour l'artiste («levé» des dimensions du lieu ou «activated surface ») et un champ d'expérience possible pour le spectateur ${ }^{26}$.

La carte et la cartographie (mapping) s'accordent en effet avec l'action artistique, au point de constituer une des fabriques spatiales de l'objet d'art-lieu. Les terrains scientifiques mobilisent les cartes et l'écriture cartographique pour découper, inventorier, mesurer, localiser, décrire, contrôler le lieu (cartes topographiques, cartes descriptives thématiques, cartes diagnostiques), et le construire dans l'objet d'art-lieu (canevas géodésiques, cartes projectives de synthèse). Sur le terrain politique, par le truchement des cartes introduites dans les négociations entre acteurs, le lieu dans toutes ses dimensions devient objet d'interlocution; par le truchement d'opérations cartographiques dont les cartes éditées constituent

\footnotetext{
${ }^{25}$ L'aerial map est une photographie aérienne verticale d'une échelle comparable aux quadrangle maps (cartes topographiques au 1/24 $000^{e}$ et au $1 / 100000^{e}$ ), sur laquelle est reportée une partie des conventions cartographiques, en particulier l'orientation et les courbes de niveau.

${ }^{26} R$. Smithson appelait à la fondation d'un Aerial Art reposant sur la transformation des sites en cartes au moyen de la transposition in situ de signes conventionnels cartographiques (voir Aerial Map Proposal for Dallas-Fort Worth Regional Airport, 1967). Il faisait aussi de la carte l'opérateur principal de sa dialectique Site/Nonsite (voir Mono Lake Nonsite, 1968 et A Nonsite, Pine Barrens, New Jersey, 1968).
} 
éventuellement le support, l'objet d'art-lieu est construit dans l'interlocution. L'acte cartographique (lecture et écriture), parce qu'il organise spatialement les énoncés séquentiels du dialogue entre acteurs, parce qu'il peut être aussi bien diagnostique que projectif, parce qu'il peut jouer des échelles et se déployer en séquences pour intégrer les temporalités du projet, réalise la liaison dynamique de l'idée d'objet et du lieu (dimensions matérielles et idéelles). La carte ici n'est pas une forme accomplie stabilisée dans un rapport d'inventaire, d'imitation ou de traduction du monde, elle est un moyen plastique de la médiation du rapport au monde et de la construction progressive d'un monde de sens dans/avec l'action artistique : elle est dotée d'une puissance performative et configurante, son référent est moins le territoire que sa re-sémantisation et sa transformation signifiante dans/avec l'action artistique. Par le truchement de la carte et par le jeu de l'acte cartographique le monde prend forme mais surtout sens pour un collectif social engagé dans l'action artistique, et celui-ci prend «ce» sens comme axe de la transformation de ce monde en objet d'art-lieu. Il n'est pas étonnant ainsi que la carte devienne une forme possible de sa mise en vue, certains objets d'art-lieu s'apparentant à des formes cartographiques (par exemple, Untitled, King County, Seattle project: Johnson Pit \# 30, 1979 de Robert Morris ; Wrapped Coast, Little Bay, Australia, One Million Square Feet, 1969, de Christo et Jeanne-Claude ; Annual Rings, 1968, et autres transpositions cartographiques d'Oppenheim) et, plus encore, à des énoncés cartographiques (micro récits situés). A propos de Umbrellas, parlant d'eux-mêmes à la troisième personne, Christo et Jeanne-Claude écrivent: "Pourquoi les parasols? Comme tous leurs projets, cette auvre d'art n'était pas seulement esthétique (...). Son but était aussi de mettre en évidence les similitudes et les différences dans le mode de vie et l'utilisation des terres par deux peuples, les plus riches du monde, vivant de part et d'autre de l'Océan Pacifique. (...) Christo et Jeanne-Claude voulaient montrer comment l'espace était utilisé différemment dans la vallée américaine et la vallée japonaise - ils avaient besoin d'un module ou d'une forme sur pied, transportable, qu'ils pourraient installer selon un schéma reflétant la disponibilité de l'espace dans la vallée d'Ibaraki et dans la vallée de Californie. »(Christo et Jeanne-Claude, $2000: 15$ ).

Enfin, la carte s'accorde aussi avec la stratégie esthétique des land artists. D'une part, l'acte cartographique est l'opérateur d'un engagement esthétique du public qui substitue l'action, comme mode de relation à l'œuvre en train de se faire, à la contemplation du sujet de la représentation fixé dans une image (le paysage). D'autre part, la carte, en tant que dispositif de réception esthétique de l'objet d'art (Ruby, 2002), est l'opérateur d'une nouvelle attitude spectatrice, une attitude distincte de celle encodée dans la perspective linéaire de la peinture de paysage et devenue habitus esthétique. Environnement esthétique ${ }^{27}$, la carte rend (techniquement et mentalement) accessible au public des lieux de l'art non réservés (souvent publics et routiniers, parfois isolés), elle contrecarre sa stratégie de possession d'un objet d'art marchandise, elle lui impose une appropriation de l'objet-lieu décentrée, en mouvement, non frontale et du dedans, faite de déambulations et de stations successives organisées dans un parcours, éventuellement de survols aériens pour le déploiement d'un point de vue zénithal et la réalisation d'une sommation cinétique de l'objet, et faite aussi d'interférences entre spectateurs.

Les opérations de terrain et cartographiques sont logiquement articulées dans la problématique foncière du Land Art. Le land claiming porte sur un sol approprié et administré, et contribue à l'extraire au moins symboliquement de son régime foncier et administratif normal, tandis que le land working l'insert dans un processus co-actoriel pour en faire le contexte et la ressource du projet d'un collectif social donné, dont l'objet d'art-lieu constitue la forme aboutie. Ces opérations ont pour effet de substituer à la valeur d'usage traditionnelle du lieu une valeur d'échange participant à la définition d'un régime postmoderne de rapport au monde (Besse, 1992), un régime présentiste de significations attachées à un lieu co-construites dans des situations dialogiques pluriactorielles et temporairement stabilisées dans l'objet d'art. En conséquence logique de leur démarche, on peut dire que les land artists tendent moins à s'approprier du sol pour poser une

${ }^{27}$ Au sens de A. Kaprow (in Chilvers, 1999 : 194), soit un espace dans lequel le spectateur est totalement inclus et impliqué par une multitude de situations. 
sculpture qui confèrera éventuellement sa valeur esthétique au lieu de sa mise en vue (problématique positionnelle de l'objet d'art ou problématique du socle), qu'à œuvrer d'art le sol pour y construire les significations collectives à la fois conditions et ressources de l'objet d'art-lieu qui en sera la manifestation forcément temporaire (problématique choraïque de l'objet d'art). Ils tendent moins à faire reconnaître par un collectif social donné un nouvel usage du sol, qu'à utiliser l'usage qu'ils en font pour en redéfinir la valeur pour ce collectif social devenu «monde de l'art », et en faire la condition/ressource de l'objet d'art dont ils ont eu le projet. Dans cette perspective, il n'est pas étonnant que l'objet d'art-lieu rassemble en lui des attributs de la carte : blanc d'une portion du township à œuvrer d'art, le land est reconstruit dans l'objet d'artlieu en un plein de sens sous la forme accomplie et fixée d'un plan cadastral forcément temporaire et d'un micro récit cartographique d'une expérience partagée de vivre-ensemble, d'une intelligence partagée d'un habiter (expérience d'être au monde porteuse de significations), d'une géographicité (dont la signification est plutôt environnementale) ou d'une territorialité (dont la signification est plutôt identitaire). Cette construction de sens, en étant donnée à voir au monde de l'art engagé dans son édification dans l'objet d'art-lieu cartographique, atteint sa monumentalité.

Certains land artists ont systématisé ce modus operandi (revendication d'un lieu pour un usage artistique, terrain et carte/cartographie), l'appliquant à des lands particulièrement denses (parcellaire de propriété, découpage sectoriel et administratif, réglementation territoriale et sectorielle, modes de propriété et d'usage du sol), l'instaurant en outil de ce qui s'apparente à de véritables projets de territoire. C'est le cas de Christo et de Jeanne-Claude qui depuis 1969 œuvrent d'art des lieux du monde occidental en proposant des idées d'objet d'art qui font lever les ressources matérielles et idéelles des lieux pour les construire dans des objets d'art qui s'apparentent à des cartes (Volvey, 2003, 2005). Leur manière de faire ne les place jamais en tant que maîtres d'œuvre sous l'empire du maître d'ouvrage (le collectif social auquel ils adressent leur projet), d'une part parce qu'ils ne répondent jamais à une commande sociale, d'autre part parce qu'ils conservent l'autonomie et la maîtrise financières du projet. Leur œuvre, dont G. Tiberghien (1995: 26) souligne qu'elle « jette sur ce moment de l'art contemporain un éclairage propre à mettre en relief certains de ses aspects », est à mon sens quasi paradigmatique du Land Art, voire d'un art du land. La définition de leur art comme «prise de possession de l'espace» se précise dans l'entretien suivant: "Traditional three dimensions sculptural space is designed by the artist. There is another space what we usually do not think about. (...) You funnel 24 hours around the clock in space designed by architects, urban planners, politicians. Space with juridictions, meanings, uses. Now what we do Jeanne-Claude and myself, we borrow that space and we create gentle disturbance for few days. (...) And we inherit everything that is inherent to that space to become a part of the work of art. We don't invent the politics of The Reichstag, we don't invent the ecology of Surrounded Islands. (...) This is why the use of the urban and rural space is so much important. (...) We are borrowing that space (...) and benefitting that space from the streets, from man made structures, from the natural forms. From the movments of the natural forms. » (in Entretien avec les artistes Volvey, 2003). D'autres land artists, à l'inverse, vont adopter des stratégies foncières qui contribuent à minimiser les enjeux propres au land claiming artistique qui caractérise le Land Art : d'une part, en intervenant dans les espaces à faible enjeu foncier soit du fait de leur substance (désert, carrières, décharges, réserves foncières des ports et aéroports, etc.), soit du fait du régime de propriété (terrains fédérés ou fédéraux de l'ouest états-unien) ${ }^{28}$, d'autre part, en faisant glisser leur intervention sous la commande publique (patronage des Counties et des Incorporated areas) ou privée (patronage d'entreprises manières ou industrielles) dans le cadre juridique

\footnotetext{
${ }^{28}$ Ils pourront à l'occasion acheter ou louer ces terrains, aboutissant ainsi leur land claiming artistique, ou bien travailler sur des terrains achetés ou loués par des institutions muséales, affaiblissant du même coup l'enjeu attaché à leur pratique outdoors. Cependant l'enjeu symbolique contenu dans cet usage artistique d'espaces périphériques, dans le contexte états-unien de la Frontier et du Land Use Reform, n'est pas négligeable.
} 
institué par le Land Reclamation ${ }^{29}$. Peu avant sa mort, au début des années 1970, R. Smithson engagea des négociations avec des entreprises impliquées dans la reconversion de leurs sites industriels et miniers ${ }^{30}$, tandis qu'en 1979 D. Oppenheim et R. Morris font partie des sept artistes invités par la King County Arts Commission (Etat de Washington, région de Seattle) à réfléchir à des projets de réhabilitation artistique de sites détériorés dans le cadre du projet «Earthworks: Land reclamation as sculpture » ${ }^{31}$. Leurs projets, qui sont alors des réponses à des commandes sociales, annoncent l'instrumentalisation du Land Art dans le marketing entrepreneurial et surtout territorial - ce dont les artistes ne sont pas forcément dupes ${ }^{32}$.

\section{Spatialités de l'art contemporain}

Dans le prolongement de ce changement de paradigme artistique opéré par le Land Art états-unien à la fin des années 1960, j'entends par Land Arts (au pluriel), l'ensemble des pratiques artistiques contemporaines dans le monde qui, quel que soit le mode d'expression qu'elles privilégient (art plastique, art sonore et musical, danse, etc.) et l'échelle de leurs interventions, œuvrent et font œuvrer le collectif social en l'abordant par une prise de possession plus ou moins subtile du lieu/de l'espace au moyen des fabriques spatiales que sont, en particulier, le terrain et la carte/cartographie, dans une visée politique. Le Land Art étasunien ainsi analysé fonctionne ici comme un schème d'intelligibilité des arts contemporains. Il ne doit pas être appliqué néanmoins comme un cadre rigide de lecture des pratiques contemporaines - d'autant plus, je l'ai dit, qu'il s'est repris lui-même sur sa radicalité -, mais considéré comme ce qui, dans un contexte plus général de mise en cause du monde de l'art institué, a expérimenté de nouveaux modes opératoires artistiques orientés vers l'action politique, apporté de nouveaux objets de pensée, impliqué un rapport au collectif social via l'action avec les lieux, pour construire dans des situations pratiques une monde de l'art pluriel et global.

En abordant la société par sa dimension spatiale les Land Arts contemporains lèvent, mettent au travail et en forme dans des objets d'art-lieux, les significations attachées aux lieux et les élaborent en dimensions temporellement et spatialement limitées d'un vivre-ensemble. Cette implication artistique contemporaine est précisément décrite dans l'article du collectif d'artistes La Luna. Sa mise en résonance avec les procédures et les objets décrits dans l'article traitant de La Pietà sud-africaine d'Ernest PignonErnest (cf. Volvey et Houssay-Holzschuch) ou dans celui présentant le travail artistique des artistes Nicolas Floc'h et Régis Perray (cf. Gilabert) montre la transversalité qu'opère la pratique contemporaine à travers les formes d'expression artistiques.

\footnotetext{
${ }^{29}$ Ainsi, Smithson, qui présentait Spiral Jetty comme « an ecological work of reclamation » et proposait : « Across the country there are many mining areas, disused quarries, and polluted lakes and rivers. One practical solution for the utilization of such devastated places would be land and water re-cycling in terms of 'earth Art'. (...) Art can become a physical resource that mediates between the ecologist and the industrialist. Ecology and industry are not one-way streets, rather they should be crossroads. Art can help to provide the needed dialectic between them. » (Cité in Beardsley, 1984 :135).

${ }^{30}$ En 1972, Smithson entama des négociations avec la Hanna Coal Company pour la reconversion de son site minier d'Egypt Valley, dans l'Ohio (Projet: Lake Edge Crescent, 1972); il entama des discussions avec la Kennecott Copper Corporation pour la reconversion d'un site d'extraction à Bingham dans l'Utah, puis avec la Minerals Engineering Company à propos de la réhabilitation de terrils sur le site de Creede, dans le Colorado (Projet : Creede, Colorado, Tailing Pond, 1972 : Garden of Tailings et Meandering Ring).

${ }^{31}$ Pour cette commande sociale, R. Morris réalisera Untitled, Earthworks for Reclaim Gravel Pit, 1979, H. Bayer, Mill Creek Canyon Earthworks Park, 742 E. Titus, Kent WA, 1979.

${ }^{32} R$. Morris a fait une analyse critique de la relation des Land artists aux programmes du Land Reclamation. Il a construit cette critique dans son objet d'art: le tronçonnage des arbres sur le bord du puit associé au surlignage des terrasses d'exploitation met formellement en exergue l'exploitation minière et rend problématique physiquement et symboliquement la jouissance scopique ou l'usage récréatif du lieu auquel pourtant son allure cartographique invite le spectateur. Un rendu qui a suscité une polémique locale (Lausson, 2008).
} 
Dans «La rue comme palette », M. Houssay-Holzschuch et moi-même faisons d'une œuvre récente du plasticien français Ernest Pignon-Ernest, La Pietà, Soweto/Warwick, mai 2002, un cas exemplaire de Land Arts contemporains. L'élaboration théorique et conceptuelle des spatialités de cette œuvre particulière, nous permet d'une part, d'évaluer les apports et les limites des concepts forgés dans les champs de l'esthétique, de l'histoire et de la critique d'art pour construire l'intelligibilité de ce type d'œuvre, et d'autre part, de promouvoir une conception relationnelle des lieux avec les choses qui les occupent. L'élaboration de cette conception chôrésique de l'œuvre d'art contemporaine dans le champ théorique de la géographie met en évidence le rôle de démarches et de procédures communes à l'art et à la géographie dans le dégagement de cette autre intelligibilité de l'art. En effet, parce que le terrain et le dessin sont les « fabriques » spatiales de $L a$ Pietà, ils fonctionnent comme les accroches et les opérateurs d'une pensée nouvelle de l'art contemporain en géographie.

D'autres pratiques artistiques ou objets d'art encore que ceux présentés par les articles ici réunis, auraient pu venir illustrer cette spatialité renouvelée de l'art contemporain, pour donner à voir la démultiplication de ses formes, ses développements du macro au micro (échelle de l'œuvre et de l'objet d'art ou ampleur de son monde de l'art), de l'action spectaculaire à la pratique discrète : l'œuvre de Christo et Jeanne-Claude bien sûr (Volvey, 2003, 2005), mais aussi les interventions environnementales que l'artiste états-unienne Julie Bargman conduit avec son D.I.R.T. Studio (Design Investigations Reclaiming Terrain), la création From/To, 2002 de Fareed Armaly présentée à la Documenta $11^{33}$, les expériences de Biomapping réalisées par l'artiste anglais Ch. Nold (Greenwich emotional map, 2005-2006) ${ }^{34}$, le Récit avec cartes du Livre blanc de Ph. Vasset (2007), ou The District Six. Public Sculpture Project, 1997 de Cape Town ${ }^{35}$. Ces œuvres d'art, qui me sont chères, sont évoquées ici comme autant d'éléments d'un corpus à constituer, à augmenter, mais aussi comme autant de pistes pour une expérience esthétique et pour une intelligence géographique de l'art.

\footnotetext{
${ }^{33}$ Texte de présentation de l'œuvre à la Documenta 11, 2002 (www.withthis.net/from/to) : «The work's central theme is the history of Palestine. Armaly (who is of Libanese-Palestinian descent) digitalized the surface of a stone, which for him is a symbol of the world and of architecture, as well as a weapon. The resulting grid pattern is pasted to the floor of the Documenta-Hall in the style of a schematic map of the Palestinian Territories. In addition, together with the Palestinian filmmaker Raschid Masharawi, Armaly presents a multimedia portrait of the region. » La documentation, présentée dans des salles reliées entre elles et à l'ensemble du dispositif par le quadrillage orienté de la carte des territoires, comprend à côté d'une exposition de cartes postales anciennes rassemblées par un anthropologue néerlandais, d'une présentation d'entretiens de femmes réalisés par une sociologue de l'EHESS dans des camps de réfugiés, de deux films documentaires (des frères Lumière, 1897, et de Tewfik Saleh, 1972), un ensemble de cartes intitulé « Israel - Palestine: cartographic manipulations », réalisé par un géographe-cartographe $\mathrm{Ph}$. Rekacewicz. La version Internet interactive de l'œuvre transforme la digitalisation de la pierre faite carte du territoire dans l'exposition, en un réseau de savoirs multidisciplinaires sur les lieux : un réseau d'articles scientifiques ou journalistiques, et de reportages dans lequel on entre par des mots-clés situés aux points carrefours des lignes.

${ }^{34}$ Extrait de la présentation du projet par l'artiste (www.biomapping.net/) : « Bio Mapping is a community mapping project in which over the last four years with more than 1500 people have taken part in. In the context of regular, local workshops and consultations, participants are wired up with an innovative device which records the wearer's Galvanic Skin Response (GSR), which is a simple indicator of the emotional arousal in conjunction with their geographical location. People re-explore their local area by walking the neighbourhood with the device and on their return a map is created which visualises points of high and low arousal. By interpreting and annotating this data, communal emotion maps are constructed that are packed full of personal observations which show the areas that people feel strongly about and truly visualise the social space of a community. How will our perceptions of our community and environment change when we become aware of our own and each others intimate body states? »

${ }^{35}$ Un projet de restitution des terres d'un quartier noir sud-africain de Cape Town, declaré zone résidentielle blanche en 1966, initié par un land claiming artistique: "The intention of the 'District Six Sculpture Festival' is to stake a claim for a public space of remembrance for the community which once lived there and was systematically removed over a period of years ending in 1981. The project has been initiated by the artists themselves, under the direction of well-known artist Kevin Brand, and has been done in consultation with a number of community organisations. » (Soudien et Meyer, 1997).
} 


\section{Les approches foncière, cartographique et scientifique dans l'art contemporain}

Dans cette perspective, j'aimerais mettre l'accent sur trois thématiques qui traversent ce numéro sans toutefois le recouper totalement et sans en épuiser la richesse, soit trois manières de poser la question de la spatialité de l'art contemporain: la question foncière/immobilière, la question cartographique, l'œuvre scientifique de l'art contemporain.

1- La question foncière est directement traitée par plusieurs articles dont les auteurs sont des géographes. Dans «Making Space for Art: the Culture Debate and the Studio Arts Movement in Hong Kong », C. Cartier propose une géographie de l'art qui soit une analyse des contextes de production, et, centrant son propos sur la production des espaces dédiés à la mise en vue de l'art dans le double contexte hongkongais d'une lutte pour l'usage de sols possédés par l'Etat et d'une planification de districts culturels par le gouvernement, elle travaille logiquement dans une perspective foncière - ici immobilière. Elle montre comment la conquête immobilière réalisée à travers la production de quartiers d'art alternatifs a été, pour les artistes qui se sont opposés aux acteurs de la planification de malls artistiques, d'une part l'outil de la définition d'un art alternatif : stratégie de différenciation (le quartier d'art alternatif devenant la condition de possibilité d'une pratique alternative de l'art et de sa reconnaissance); d'autre part l'outil de la définition d'un art hongkongais postcolonial: stratégie de territorialisation. Le contre-art s'édifie dans la conquête immobilière et à travers la production d'espaces dédiés à l'art (production, mise en vue, diffusion d'objets d'art) où se joue un art de la rencontre avec le public condition de sa valeur/signification identitaire.

Adoptant une perspective similaire, l'article de Ch. Ambrosino - «La monstration de la ville contemporaine. L'exemple d'Hoxton à Londres et du mouvement Young British Artists dans les années $1990 »-$ propose une analyse de la conquête du quartier d'Hoxton (Londres) et de son branding par les artistes. L'auteur montre comment la conquête foncière/immobilière de l'ancien quartier ouvrier d'Hoxton par des artistes-entrepreneurs sous la forme des artist-run spaces, conquête qui reposait sur la mobilisation d'un ensemble de ressources spatiales matérielles (friches et de locaux industriels disponibles, bas prix du foncier), avait favorisé la levée d'une ressource spatiale idéelle (culture ouvrière contestataire) et sa primo-élaboration par les artistes dans une stratégie identitaire par différenciation: la participation à la conquête foncière signalait à la fois, pour chacun des artistes, sa pratique alternative de l'art et, pour tous, l'appartenance à un mouvement d'avant-garde. Mais l'article va au-delà, en montrant comment la mobilisation des attributs idéels du quartier s'organise en un micro récit sur le lieu, micro récit mobilisable à son tour comme ressource symbolique pour un projet artistique global : le branding artistique du quartier d'artistes. L'intégration in fine de ce branding artistique dans une stratégie de promotion immobilière du quartier devenu élément de la City Fringe, non seulement boucle, mais retourne l'enjeu foncier des Land Arts.

Dans «La fin des ateliers ou le portrait de l'artiste nomade», T. Gilabert centre son propos sur l'évolution récente de la géographie des lieux de création de l'art en France en tenant ensemble deux paramètres : l'évolution des pratiques et l'évolution du marcher immobilier. Il fait de l'évolution du marcher immobilier à la hausse et de l'échec de la compensation de celle-ci par des politiques culturelles publiques, le contexte d'une sortie hors les murs de l'art français, du développement consécutif d'une esthétique relationnelle ou d'un art participatif, du développement de sa double dimension nomade et mondiale, sous la forme de chantiers artistiques mobiles. La pratique de balayage des sols de l'artiste français R. Perray, par sa discrétion même et par la simplicité abstraite du geste qui lui est lié, signale de manière quasi emblématique l'enjeu foncier des Land Arts contemporains.

De son côté, le texte du philosophe Ch. Ruby - «Variations sur les espaces et les lieux de l'art »qui aborde l'art public en interrogeant d'abord sa relation d'évidence au lieu, en réfléchissant ensuite à la manière dont le lieu est fait lieu dédié à l'art, rencontre la question de la multidimensionnalité (matérielle, idéelle) de l'espace et la pose comme préalable à une compréhension de ce qui est public dans l'art. Une 
définition non physicaliste de l'espace, lui permet de rattacher la question de la signification (politique) de l'art public au lieu.

2- La question de la carte et de la cartographie est abordée directement ou indirectement par plusieurs auteurs d'origine disciplinaire diverse traitant plus pour certains de la spatialité des objets d'art que de celle des pratiques artistiques. Elle entre en résonance avec des textes qui reviennent sur les différents moments de la spatialité dans les arts pour déconstruire la perspective linéaire ou en penser les alternatives. Dans cet ensemble de textes, la référence directe ou indirecte à la carte est moins affaire de représentation que de mode de spatialisation : la carte ou le cartographique est, en particulier, ce par quoi l'art traite ou rend possible le mouvement et l'articulation du temps et de l'espace.

A. Lacan, «Joao Fiadeiro I am here : cartographie d'un parcours sensible », pense la logique spatiale d'une chorégraphie particulière sur le modèle de la cartographie et la danse sur le modèle de la carte. Après avoir conduit, en l'étayant sur des citations de J. Lévy, une réflexion sur la carte comme dispositif d'écriture/lecture, réflexion à travers laquelle elle fait du rapport image/texte (légende) la manifestation d'une lacune de la représentation et de l'expérience de lecture la condition de son dépassement, l'auteure montre comment la pièce qu'elle étudie rapproche la chorégraphie, en tant qu'écriture lacunaire d'une spatialité, de la cartographie (en l'occurrence un bodymapping) et l'expérience spectatrice, en tant que comblement de la lacune dans l'expérience mentale de déplacement, de la lecture de carte. L'ensemble fonctionne par le truchement d'un tracé au sol, sorte de relevé topographique de la danse (du parcours dansé), devenu aprèscoup support matériel de l'expérience spectatrice.

Dans «Le cinéma et la raison cartographique des images », T. Castro se réfère aux textes de J. B. Harley, F. Farinelli et Ch. Jacob pour mettre en évidence plusieurs formes de la raison cartographique (atlas, panorama, vue aérienne, etc.) dans le cinéma des origines. Des formes qui instaurent dans l'écriture filmique les conditions de possibilité de la spatialisation du temps et du mouvement, et d'une expérience spatiale du monde, celle du regard cartographique. Dans cette archéologie d'œuvres cinématographiques, l'auteure élabore une matrice théorique pour penser le «tournant spatial » du cinéma contemporain et de son étude.

M. Gérardot, dans «Robert Delaunay, le rythme et la géographie », propose de mobiliser les compositions géométriques de l'artiste pour participer à l'élaboration d'un concept de rythme en géographie à travers lequel elle conçoit la possibilité d'articuler la temporalité et la spatialité des phénomènes. On lit à travers ses lignes, et dans sa référence à R. Court (1992), combien la question de l'écriture du rythme (Court parle de «picturalisation du musical»), son rendu graphique dans un double jeu de rapports points/lignes/surfaces et forme/fond/cadre, est à l'origine de la spatialisation d'un concept lié à la temporalité. C'est peut-être moins le rythme que l'écriture du rythme, parce qu'elle le spatialise et le met en perspective du modèle cartographique, qui ouvre cette possibilité d'articulation du temps et de l'espace en géographie.

J. Monnier rattache «La géographie de Jacques Villeglé » à sa stratégie de «décentralisation ». Au fondement d'une pratique de terrain reposant sur la déambulation, l'observation et le prélèvement de parcelles d'affiche dans les «emplacements réservés » de l'encollage sauvage urbain, cette décentralisation rappelle l'outdoors et les enjeux fonciers décrits ci-dessus, tandis que le découpage de la peau des murs rappelle l'acte de levé des dimensions du lieu propre à la stratégie d'in situ. Le marouflage des échantillons prélevés sur la toile, l'intitulé toponymique (nom du lieu d'encollage et date du prélèvement), l'accrochage aux murs des musées font de ces «affiches lacérées » et de leur assemblage un dispositif de transfert de données urbaines du site (urbain) vers le nonsite (muséal) - pour reprendre les termes de R. Smithson -, site dont elles documentent la dimension politique, sur un mode métonymique. Si l'auteure travaille ces affiches à partir de la notion de paysage mais en dehors de la problématique de la représentation, on voit aussi combien l'arbitraire du cadre qui découpe l'information et permet l'assemblage des informations sur le lieu, la bidimensionnalité, le jeu d'échelles, la transparence référentielle, instaurent la figure de la carte urbaine (du plan) en clé de lecture de ces assemblages de fragments d'urbanité. 
Le tournant cartographique dans les arts et l'analyse esthétique contemporains ne va pas sans que soit pensée de manière critique la perspective linéaire et le paysage, pour poser les paradoxes et les limites de ce mode de spatialisation, pour envisager d'autres modes d'accès à la représentation et à l'imaginaire géographique.

C. Simond, dans «Les pérégrinations de l'espace. L'ange de Patrick Bokanowski (1982)», analyse la critique cinématographique de l'espace perspectiviste conduite par le cinéaste dans son film : la difficile conciliation du mouvement et du temps (instant/durée) avec cette forme de spatialisation, la problématique de l'enfermement spatial. Une analyse qui n'est pas sans écho avec celle conduite par T. Castro.

O. Melendez, dans «L'imaginaire géographique mis en perspective par l'anamorphose », propose à la réflexion des géographes sur l'imaginaire géographique cette technique artistique de dissimulation d'image dans l'œuvre et de dévoilement de celle-ci par un procédé optique ou géométrique. Moyen d'un art contemporain (Marcus Raetz) qui met en jeu les habitudes spectatrices d'une construction frontale et objective du réel, elle offre un autre espace de projection pour un autre rapport de construction entre perçu et réel, et partant pour l'expérimentation de l'imaginaire géographique.

La mise en perspective des textes d'O. Bratosin et de M. Scarpulla est intéressante en ce que d'une part, elle témoigne du parcours croisé de la géographie et des arts autour d'une notion commune, le territoire une notion liée à la carte comme au paysage -, et d'autre part elle donne à voir ce qui est en transformation dans l'approche spatiale des arts aujourd'hui. Dans la tradition de la géographie sociale, c'est bien le texte littéraire comme source pour accéder à l'idéalité des lieux - l'imaginaire/représentation du territoire - et la représentation paysagère comme mise en forme stabilisée de celle-ci, qu'O. Bratosin travaille dans «Le Baragan: espace géographique dans la littérature roumaine». La résonance de ce texte avec celui d'O. Melendez s'établit autour des différents modes de spatialisation qui opèrent et médiatisent chacun à leur manière la représentation de l'idéalité attachée à l'espace. Tandis que, dans «Danses d'exil. Les chorégraphies contemporaines d'une identité étrangère face aux terres habitées par l'artiste et sa compagnie », c'est à travers la pratique chorégraphique et la danse (la situation dansée) que M. Scarpulla aborde le discours identitaire de l'art contemporain, tel qu'il se construit en dehors d'une politique de représentation. Son étude croisée de trois pièces montre comment un là-bas, d'où l'artiste est exilée, et un ici, où le lieu et la danse composent une situation artistique instaurée en condition et ressource d'une construction identitaire inédite, s'articulent au moyen d'un terrain - qui opère par prélèvement matériel ou photographique d'objets, de fragments sonores ou gestuels et même de personnes -, et d'une chorégraphie - qui élabore, via la collection et le collage de citations, le matériau prélevé là-bas et le cadre scénographique d'ici. La situation est facilitatrice d'un processus de territorialisation - l'auteur parle du plateau comme d'un «territoire d'exil»dans le mouvement duquel se construit une «identité étrangère » alors transmissible, reconnaissable et éventuellement partageable avec le spectateur dans l'expérience esthétique.

3- Plusieurs articles d'origine disciplinaire différente tirent les conséquences théoriques de la mise en évidence des nouvelles dimensions spatiales des pratiques artistiques contemporaines pour affirmer que l'art contemporain participe aujourd'hui au concert des disciplines, voire des sciences, de l'espace, et pour interroger en retour le statut opératoire ou scientifique des sciences traditionnelles de l'espace : l'architecture et la géographie.

Avec «Parce que le monde n'est pas un cube blanc... », le collectif d'artistes La Luna propose un texte exemplaire sur les formes discrètes des Land Arts contemporains, quand il rend compte des micro procédures par lesquelles se crée «la relation dialogique de la forme artistique à la sphère publique »-le «campement urbain» ou l' "espace à vivre» conçu comme un espace de captation et d'élaboration de matières sources -, ou quand il présente son projet En fait c'est pas si loin, Saint-Herblain, 2002-2003 $3^{36}$. Mais

${ }^{36}$ On verra d'ailleurs comme une manifestation de plus du tournant spatial de l'art contemporain, du renouvellement pluridisciplinaire qui lui est associé et de la part que peut y prendre la géographie, le fait que ce projet s'inscrit dans le cadre de «Passe-Partout», un 
son horizon s'ouvre encore quand, définissant l'objet d'art-lieu comme «laboratoire de sensibilisation » ou « cellule de veille », les artistes/auteurs énoncent leur ambition de participer à la formation d'un savoir critique empirique pluridisciplinaire sur la ville, base opérationnelle de la construction de l'urbanité contemporaine.

C. Aventin, dans «Les arts de la rue pour observer, comprendre et aménager l'espace public », développe une analyse phénoménologique des arts de la rue conduite du point de vue de l'architecte. Elle fait des événements artistiques qui viennent perturber le rapport à la ville, des outils d'une prise de conscience de l'ordinaire urbain par ses usagers/résidents/administrateurs. Mais au-delà elle intègre les arts de la rue au champ des sciences de l'ingénierie spatiale, aux côtés de l'aménagement urbain et l'urbanisme, au titre d'un matériau spatial et d'une visée sociale communs. Elle met en avant les registres communs de la pratique artistique et aménageuse : circulation des termes servant à désigner les pratiques, partage des codes et des savoirs pratiques de maitrise des contextes d'action, association dans des programmes. Si elle reconnaît une plus grande compétence procédurale des (architectes) aménageurs/urbanistes, elle souligne la compétence sociale des artistes de rue, pour en faire l'élément de leur exemplarité contemporaine. Elle reconnaît aussi dans la temporalité propre aux arts de rue le moyen de tests grandeur nature pour les opérations d'aménagement ou d'urbanisme conçues par les architectes pour durer.

E. Boutouyrie, dans «L'art urbain comme pensée et pansement de la ville en mouvement : un regard à partir de deux publications aux accents géographiques », montre la performativité de l'art contemporain par rapport à la mise en évidence et à la mise au travail réflexif de notre urbanité contemporaine. L'auteur met l'accent sur la pertinence de cette intelligibilité produite par l'art quand, délocalisé, il traite des espaces périphériques ou virtuels de l'urbanité contemporaine.

H. Regnauld et Ch. Viart s'intéressent aux «Fragments géographiques », un texte interdisciplinaire qui explicite un projet muséal à l'origine duquel ils se trouvent. Centrant leur réflexion sur la méthode de terrain (procédures, techniques), ils font des artistes contemporains des praticiens de l'espace au même titre que les géographes. Au-delà, s'intéressant aux rapports entre pratique/procédures-techniques/construits et les plaçant dans la perspective d'une ontologie réaliste (du visible) supposée partagée par les géographes et les artistes, ils interrogent le statut respectif de la mémoire, de l'exactitude et de l'erreur dans les processus de création artistique et scientifique, et partant le régime de validité de leurs objets spatiaux respectifs. Un tel positionnement épistémologique - qui se déploie des pratiques spatiales vers leurs construits -, permet aux auteurs d'interroger de manière croisée d'une part, le régime de scientificité de la géographie et d'autre part, le régime de fiction des arts.

\section{Bibliographie}

\section{Sources primaires}

BEERN W. A. L. (1971), «From Exhibition to Activity (introduction), Sonsbeek 71, Catalogue de l'exposition, 19 juin $\quad-\quad 15 \quad$ août $1971, \quad$ Arnhem. http://www.experimentaltvcenter.org/history/people/ptext.php3?id=84\&page=1, accédé en 2008.

BRAYER M.-A. (2000), Orbis terrarum. Ways of worldmaking / Cartography and contemporary Art, catalogue d'exposition, Musée Plantin-Moretus and Surrounding juin-septembre 2000, GandAmsterdam, Ludion éd.

projet d'exploration territoriale introduit par une expression empruntée au géographe R. Pourtier qualifiant sa pratique de terrainfabrique spatiale de la science géographique -, soit le "ça-voir». 
CHRISTO et JEANNE-CLAUDE (2000), Les erreurs les plus fréquentes, Paris, éd. Janninck.

DIAMONSTEIN B. (1979), Inside New York's Art World, NY, Rizzoli.

«Discussion with Heizer, Oppenheim, Smithson », in Avalanche, automne 1970 [Repris dans N. Holt (éd.), The writings of Robert Smithson, NY, New York University Press, 1979; et en Annexe dans Kastner et Wallis, 1998: 201-205. Trad. in Tiberghien, 1995: 277-283].

Los Angeles Times, «Interview with Christo », édition du 23 mai 1991.

SOUDIEN C. et MEYER R. (eds) (1997), The District Six Public Sculpture Project, Catalogue de l'exposition, Cape Town, District Six Museum, s.d.

VASSET Ph. (2007), Un livre blanc. Récit avec cartes, Paris, Fayard.

YANAGI M. (1989), «Interview de Christo », in Catalogue d'exposition, Christo from the Lilja Collection, Musée d'art Moderne et contemporain, 4 juillet - 30 septembre 1989, Nice, Editions des Musées de Nice.

\section{Entretiens}

VOLVEY A. (2003), Entretiens avec Christo et Jeanne-Claude, New York, juillet 2003.

\section{Sources secondaires}

BEARDSLEY J. (1984), Earthworks and Beyond. Contemporary Art in the Landscape, NY, Abbeville Press Publisher.

BECKER H. (1988), Les mondes de l'art, Paris, Flammarion (éd. originale 1982).

BERQUE A. (2003), «Lieu», in Lévy J., Lussault M. (dir.), Dictionnaire de la géographie et de l'espace des sociétés, Paris, Belin.

BESSE J.-M. (1992), «Entre modernité et postmodernité : la représentation paysagère de la nature », in M.-C. Robic (dir.), Du milieu à l'environnement, Paris, Edition Economica, pp. 89-121.

BESSE J.-M. (2000), Voir la Terre. Six essais sur le paysage et la géographie, Arles/Versailles, Actes Sud/ENSP.

BLANC N. (2009), Vers une esthétique environnementale, Editions Quae.

BOUTOUYRIE E. (2006), Artialisation et ontogenèse des lieux contemporains. Etude du courant musical psychedelic trance, Thèse de doctorat de l'Université de Paris 1-Sorbonne.

BRAYER M.-A. (1995), « Mesures d'une fiction picturale : la carte de géographie », in Exposé. Revue d'esthétique et d'art contemporain, $\mathrm{n}^{\circ} 2$, pp. 7-23.

BRIOT M.-O. et Humblot C. (éds) (1980), La peau des murs, Paris, Limage.

BROSSEAU M. (1996), Des romans-géographes, Paris, L'Harmattan.

Les Cahiers d'EspacesTemps (2002), «A quoi œuvre l'art ? », n78-79.

CANT S. G., MORRIS N. J. (dir.) (2006), «Geographies of art and the environment», in Social \& Cultural Geography, Volume 7, Issue 6 December.

CASTRO T. (2008), «Les cartes vues à travers le cinéma », in Textimage, $n^{\circ} 2$, «Cartes et plans ». http://www.revue-textimage.com/03_cartes_plans/castro1.htm 
CHANG T. C., Lee W. K. (2003), « Renaissance City Singapore : a study of arts spaces », in Area, vol. $35, \mathrm{n}^{\circ} 2$, pp. 128-141.

CHANG T. C. (2008), «Art and Soul : powerful and powerless art in Singapore », in Environment and planning A, vol. 40, pp. 1921-1943.

CHILVERS (1999), A Dictionary of Twentieth-Century Art, New York, Oxford University Press.

CORBEL L. (2008), «Entre cartes et textes : lieux et non-lieux de l'art chez Smithson », in Textimage, «Cartes et Plans », $\mathrm{n}^{\circ} 2$. http://www.revue-textimage.com/03_cartes_plans/corbel1.htm

COURT (1992), Rythme musical et forme esthétique», in J.-J. Wünenburger (dir.), Les rythmes. Lectures et théories, Paris, L'Harmattan / Centre culturel international de Cerisy.

DACOSTA KAUFMANN T. (2004), Toward a Geography of Art, Chicago, University of Chicago Press.

DE SOTO H. (2005), Le mystère du capital : pourquoi le capitalisme triomphe en Occident et échoue partout ailleurs, Paris, Flammarion.

DEBARBIEUX B. (1995), «Imagination et imaginaire géographiques », in Bailly A. et al. (dir.), Encyclopédie de la géographie, Paris, Economica ( $2^{\mathrm{e}}$ éd.).

GARRAUD C. (1994), L'idée de nature dans l'art contemporain, Paris, Flammarion.

GILABERT (2004), Géographie de l'art contemporain en France, Thèse de géographie soutenue à l'Université de Nantes.

GRISON L. (2002), Figures fertiles : essai sur les figures géographiques dans l'art occidental, Paris, éd. J. Chambon.

GRISON L. (2005), Les stries du temps : l'artiste, le lieu et la mémoire. Collages, Paris, éd. Champ social.

GUIU C. (2009), « Les géographies sonores : rythmes et contrepoints », in Y. Raibaud (dir.), Comment la musique vient-elle au territoire ?, MSHA, Bordeaux (à paraître).

KASTNER J., WALLIS B. (1998), Land and Environmental Art, Londres, Phaïdon Press.

HEIDEGGER M. (1996), Die Kunst und der Raum / L'Art et l'Espace, St Gallen, Erker-Verlag (1 ${ }^{\text {ère }}$ éd. franco-allemande, 1969).

Hérodote (1987), « Paysages en action », $\mathrm{n}^{\circ} 44,1^{\mathrm{er}}$ trimestre 1987.

KRAUSS R. (1979), « Sculpture in the Expended Field», in October, printemps 1979.

MILES M. (1997), Art, Space and the City : Public Art and Urban Futures, Londres et NY, Routledge.

MINTY Z. (2006), «Post Apartheid Public Art in Cape Town: Symbolic Reparation and Public Space », in Urban Studies, vol. 43, n², pp. 421-440.

MONDADA L. (2000), «Pratiques discursives et configuration de l'espace urbain », in J. Lévy, M. Lussault (dir.), Logiques de l'espace, Esprit des lieux. Géographies à Cerisy, Paris, Belin, pp. 165-175.

LAUSSON A. (2008), «L'enjeu écologique dans le travail des Reclamation Artists », hal.archivesouvertes.fr/docs/00/33/77/27/PDF/3.Lausson.pdf

MALDINEY H. (1973), Regard, parole, espace, Lausanne, L'âge d'homme.

PENDERS A.-F. (1995), Conversations avec Christo et Jeanne-Claude, Gerpinnes, Editions Tandem. 
PENDERS A.-F. (2000), En chemin. Le Land Art, (T1) Partir / (T2) Revenir, Bruxelles, Lettre Volée éd.

PINDER D. (2005), «Arts of Urban Exploration », in Cultural Geographies, vol. 12, n4 , pp. 383-411.

REGIMBALD M. (2008), «Quand l'art découvre ses cartes », in Textimage, « Cartes et Plans », n². http://www.revue-textimage.com/03_cartes_plans/regimbald1.htm

REGNAULD H. (1998), L'espace : une vue de l'esprit ?, Rennes, PUR.

ROGER A. (1978), Nus et paysages. Essai sur la fonction de l'art, Paris, Aubier Montaigne.

RUBY Ch. (2002), «Esthétique des interférences », in Les Cahiers d'EspacesTemps, «A quoi œuvre l'art?», n78-79, pp. 8-21.

SOMDAHL-SANDS K. (2006), «Triptych : dancing in Thirdspace », in Cultural Geographies, $n^{\circ} 13$, pp. 610-616.

STASZAK J.-F. (2006), Gauguin voyageur. Du Pérou aux îles Marquises, Paris, éd. Solar.

STRAUS E. (1989), Du sens des sens. Contribution à l'étude des fondements de la psychologie, Grenoble, J. Million, (Trad. française. Ed. allemande, 1935).

Textimage (2008), « Cartes et Plans », $\mathrm{n}^{\circ} 2$.

TIBERGHIEN G. (1995) Land Art, Paris éd. Carré.

TIBERGHIEN G. (1996) Land Art Travelling, Valence, ERBA.

TIBERGHIEN G. (2001), Nature, Art, Paysage, Arles/Versailles, Actes Sud/ENSP.

TIBERGHIEN G. (2007), Finis Terrae. Imaginaires et imaginations cartographique, Paris, Bayard.

VANCI-PERAHIM M. (dir.) (2006), Atlas et territoires du regard. Le géographique de l'histoire de l'art (XIXe et XXe siècles), Paris, Publications de la Sorbonne, Actes de Colloque.

VAN ESSCHE E. (dir.) (2007), Les formes contemporaines de l'art engagé. De l'art contextuel aux pratiques documentaires, Bruxelles, La Lettre Volée.

VOLVEY A. (2002), «Fabrique d'espaces : trois installations de Christo et Jeanne-Claude», in Les Cahiers d'EspacesTemps, numéro spécial A quoi œuvre l'art ? Esthétique et espace public, $\mathrm{n}^{\circ} 78$ 79, pp. 68-85. Texte republié in Revue Roumaine d'Histoire de l'Art (série Beaux-Arts), Tome 38, 2001 (paru en 2003).

VOLVEY A. (2003), Art et spatialités d'après l'œuvre in situ outdoors de Christo et Jeanne-Claude. Objet textile, objet d'art et œuvre d'art dans l'action artistique et l'expérience esthétique, Thèse de Doctorat de l'Université de Paris I-Sorbonne.

VOLVEY A. (2005), «Christo et le Land Art. Dans la carte du territoire la monumentalité christolienne », in Ligéia. Dossiers sur l'art, numéro spécial Brancusi et la sculpture, n $57-58-59$ 60, janvier-juin 2005, pp. 221-232. 